\title{
Multiple objective optimal control of integrated urban wastewater systems
}

\author{
Guangtao Fu*, David Butler, Soon-Thiam Khu \\ Centre for Water Systems, School of Engineering, Computer Science and Mathematics, \\ University of Exeter, North Park Road, Harrison Building, Exeter EX4 4QF, UK \\ ${ }^{*}$ Corresponding author. Tel: +44 (0)1392 264075 Fax: +44 (0)1392 217965 \\ Email addresses: G.Fu@exeter.ac.uk; \\ D.Butler@exeter.ac.uk; \\ S.T.Khu@exeter.ac.uk
}

\begin{abstract}
Integrated modelling of the urban wastewater system has received increasing attention in recent years and it has been clearly demonstrated, at least at a theoretical level, that system performance can be enhanced through optimized, integrated control. However, most research to date has focused on simple, single objective control. This paper proposes consideration of multiple objectives to more readily tackle complex real world situations. The water quality indicators of the receiving water are considered as control objectives directly, rather than by reference to surrogate criteria in the sewer system or treatment plant. A powerful multi-objective optimization genetic algorithm, NSGA II, is used to derive the Pareto optimal solutions, which can illustrate the whole tradeoff relationships between objectives. A case study is used to demonstrate the benefits of multiple objective control and a significant improvement in each of the objectives can be observed in comparison with a conventional base case scenario. The simulation results also show the effectiveness of NSGA II for the integrated urban wastewater system despite its complexity.
\end{abstract}

Keywords: Integrated modelling; Multiple objective optimization; NSGA II; Optimal control; Urban wastewater system 


\section{Introduction}

There is an increasing awareness of the need to view the urban wastewater system as a whole to include at least the sewer system, wastewater treatment plant and receiving water body. This was initially driven by the motive to achieve improved system performance in design or operation, measured by receiving water quality indicators, and therefore a better receiving water environment (Rauch et al., 1998). In the recent years, the need for such an integrated system has been further inspired by the EU Water Framework Directive (WFD), which aims to achieve 'good' ecological and chemical status in all water bodies by 2015 (CEC, 2000).

Only since the 1990s, has integrated modelling of urban wastewater systems been made possible with the development of simulation models for each individual sub-systems, improved computing capacity, and holistic environmental legislation (Butler and Schütze, 2005). Since then, the benefits of integrated modelling, particularly with regard to real time control, have been well addressed and case studies reported in the literature (for example, see Schütze et al., 2002; Schütze et al., 2004; Zacharof et al., 2004; Vanrolleghem et al., 2005). Butler and Schütze (2005) demonstrated theoretically that a significant improvement in performance of the system can be achieved by integrated real time control, even in cases for which local control does not appear promising

Optimal control of the urban wastewater system aims to derive a control strategy to achieve the best system performance with respect to various criteria (control objectives). Traditionally, these objectives lie in the sewer system or treatment plant due to the limits of separation in modelling of each individual sub-system, such as minimizing combined sewer overflow (CSO) volume or frequency, and maintaining treatment plant effluent standards. With the development of integrated models, it is now possible to directly use water quality parameters of the receiving water body as control objectives. Many different indicators may be considered to measure water quality status depending on potential 'use' of the receiving water. For example, within the WFD, chemical status refers to a 'priority list' of pollutants, and ecological status is assessed by considering biological, physico-chemical, and hydro-morphological elements, although the relationships between them 
are still not fully understood. Economic factors will have to be taken into account in real world applications of control.

Though optimal control has received much attention in the context of integrated modelling (for a review, see Schütze et al., 2004), most researchers have focused on single objective optimization. For example, Rauch and Harremoës (1999a) considered overflow volume and the mean dissolved oxygen (DO) concentration level calculated by a moving average as separate objectives, and a tradeoff between the two objectives was found when comparing the two single objective optimization runs. In this situation, however, only two solutions were found and the whole tradeoff curve was not derived. Schütze et al., (2002a) introduced various objectives based on DO and ammonium concentration, however, only a single objective was chosen in the optimization process. In Vanrolleghem et al. (2005), ammonia concentration was chosen as the objective to derive the optimal control strategy. However, in practice, a control strategy will be required to meet multiple and possibly conflicting objectives, in order to meet the many needs placed on receiving waters. It is unlikely that all objectives will reach their optimum values simultaneously, and only a set of Pareto-optimal solutions can be derived where one objective cannot improve without the reduction of at least one of the others. Therefore optimal control of the urban wastewater system is actually a multiple objective optimization problem where tradeoffs between objectives must be made to obtain a satisfying overall performance in terms of all the considered objectives. Application of multiobjective optimization methods has been introduced by Schütze et al. (2002b), but discussions of the tradeoffs were limited.

The genetic algorithm has proven to be a very powerful technique to find optimal solutions for many real-world optimization problems, and it has been widely applied in the field of water and wastewater engineering (Savic \& Khu, 2005; Khu et al, 2006). The benefit and potential of application of genetic algorithms in urban wastewater systems were discussed by Rauch and Harremoës(1999b) and Boomgaard et al. (2001). Schütze (1998) has shown that genetic algorithms can be successfully applied to real time control optimization. Rauch and Harremoës (1999a) also applied genetic algorithms to developing optimal real time control strategy in terms of water quality in the receiving water. For multi-objective control optimization, however, no 
substantial applications have been reported in the literature although the potential has been demonstrated by Rauch and Harremoës (1999b) and Muschalla et al. (2006).

This paper aims to investigate the optimization of multi-objective control of urban wastewater system using genetic algorithms. Compared with a single objective solution, a set of Paretooptimal control strategies can be identified instead of a single one. This will allow the decision maker to have a more complete knowledge of the tradeoff structure between conflicting objectives when making a decision. The chosen optimization method is the non-dominated sorting genetic algorithm (NSGA II), and a brief introduction to it is given in Section 3. A case study is used to demonstrate this method, and two objective and three objective scenarios are analyzed to illustrate the benefits of multi-objective control.

\section{Integrated modelling}

Detailed reviews of integrated modelling have been produced by Rauch et al. (2002) and Schütze (1998) and will not be repeated. However, below is a brief description of the integrated model used in this study.

The integrated model was developed using the SIMBA5 simulation tool, developed and marketed by IFAK (2005). This tool is produced in the MATLAB/SIMULINK environment, which allows users to develop their own modules for their specific needs. The integrated model is structured to consist of three simulation sub-models for simulating the water quantity and quality processes in the sewer system, treatment plant and river, i.e., KOSIM for the sewer system (ITWH, 1995), Activated Sludge Model No.1 (ASM1) for the treatment plant (Henze et. al. 1986), and EPA storm water management model (SWMM5) for the river (Huber and Dickinson, 1988). Additionally, some blocks for the interactions between the three sub-models are also included in the model because of the different state variables used in the sub-models.

\subsection{Sewer system}

The processes considered in KOSIM include surface runoff and wash-off, flow and pollutant transport in sewers, and storage tanks. In the surface runoff, different processes are simulated for impervious and pervious catchment areas. For the former, wetting, depression storage, and 
evaporation losses are taken into account, and for the latter, a modified Horton approach for longterm simulation is used for modelling infiltration (Paulsen, 1986). Flow transport within a subcatchment is simulated by a simple cascaded linear reservoir model and flows between subcatchments are routed by translation only. Pollutants arise from two sources: domestic dry weather flow and rainfall runoff, and the former is in diurnal and weekly patterns. The pollutant concentrations in the two sources are considered as constant values. The pollutants are assumed to be completely mixed in the system without any biochemical transformations. Pollutants simulated in the sewer system are suspended solids, volatile suspended solids, total COD, soluble COD, ammonium and nitrate. Storage tanks of different types are also considered and these can be controlled via outlet pumps.

Simplicity in water quantity and quality modelling of the sewer system is important in integrated modelling; it reduces simulation time to a greater extent, and therefore makes it more practical for real time control optimization, which generally requires thousands of model runs.

\subsection{Wastewater treatment plant}

The treatment plant consists of a storm tank, primary clarifier, and activated sludge reactor and secondary clarifier. ASM1 was chosen to model the biological processes, and is generally accepted as state of the art in simulating municipal activated sludge wastewater treatment plants (Henze et. al. 1986). It has been included in many simulation platforms for wastewater treatment process and control optimization, and is included in the Benchmark Simulation Model No.1 (Copp, 2002). In the integrated model, several processes in the original ASM1 have been modified to make it more flexible and adaptable to various situations, for example, the low-loaded plants where all ammonia may be nitrified or incorporated, resulting in concentration below zero (IFAK, 2005). The settling processes are modelled by the double exponential settling velocity function of Takács et al. (1991) because of its international acceptability.

\subsection{River model}

SWMM5 is one of the most advanced computer models for simulation of hydrodynamic water and pollutant transport in sewer systems. SIMBA5 makes use of the SWMM5 executable as a block to simulate the sewer system and river. This enables a fully dynamic simulation of river flow, 
including backwater effects. Biochemical transformation processes may also be represented by integration of a water quality model in Petersen matrix notation. Pollutants considered include BODr and BODs (readily and slowly biodegradable fractions), total ammonia and dissolved oxygen. Discharges into the river include CSOs from the sewer system, storm tank overflows and treatment plant effluent.

Due to the different sets of pollutants used in the three sub-systems, modules are used to convert between the subsystems. All of the subsystems run in a synchronous way and this makes completely integrated control of the whole system possible.

\section{Multiple objective optimization}

\subsection{Problem formulation}

A control strategy for urban wastewater systems can be described by $n$ controllable parameters:

$$
\mathbf{x}=\left[x_{1}, \quad x_{2}, \cdots, x_{n}\right]
$$

Thus, the multi-objective control problem can be stated as the following optimization problem:

$$
\operatorname{Min} F(\mathbf{x})=\left\{f_{1}(\mathbf{x}), \cdots, f_{m}(\mathbf{x})\right\}, \quad \mathbf{x} \in \Omega
$$

Subject to

$$
\begin{gathered}
g_{i}(\mathbf{x}) \geq 0 \\
h_{j}(\mathbf{x})=0
\end{gathered}
$$

Where $\Omega$ is the feasible solution space, and $f_{1}, \cdots, f_{m}$ are the $m$ objective functions to be

simultaneously minimized. $g_{i}(\mathbf{x})$ is the inequality constraints, $i=1, \ldots, k$, and $h_{j}(\mathbf{x})$ is the equality constraints, $j=1, \ldots, K$. Generally speaking, the objectives are non-commensurable, so they cannot be compared with each other directly.

Definitions of the objective functions usually involve water flow and quality states in the system, so their evaluations can only be achieved through the integrated model. A simplified representation of the objective functions (for example, linearization) seems to be problematic for the integrated system due to its complexity (Schütze et al., 2002b). In this situation, a global 
optimization method is most appropriate and therefore a multiple objective genetic algorithm is applied in this research.

\subsection{Algorithms}

NSGA II is an effective method for multiobjective optimization developed by Deb et al. (2002). It has three significant features: (i) using an efficient non-domination sorting scheme so that the computational complexity is reduced to a great extent; (ii) eliminating the sharing parameter, and (iii) using an elitist selection method that significantly speed up the capture of Pareto surfaces. Background information and thorough descriptions of Pareto optimization and genetic algorithms, can be found in Deb (2001), and Coello Coello et al. (2002).

In NSGA II, the concept of Pareto-dominance is used to rank the individuals (control strategies) of a population. For example, an individual $d_{1}$ dominates another $d_{2}$ if and only if it performs as well as $d_{2}$ with regard to all objectives and strictly better in at least one objective. The nondomination sorting approach divides the population into different ranks. The individuals which do not dominate each other but dominate all the others in the population are assigned rank 1 , the fittest individuals in the population. Amongst the remaining individuals in the population, the individuals which do not dominate each other but dominate all the others are assigned rank 2 . The same procedure is repeated until all the individuals are assigned a rank. Besides the nondomination rank, crowding distance of each individual is also calculated by the average Euclidean distance between the individual and those adjacent individuals in terms of each of the $m$ objectives. A binary tournament selection is employed where two individuals are selected at random from the population and the fitter one is selected as a parent. The selection process at various stages of this algorithm is based on two criteria: non-domination rank and crowding distance. First and foremost is the rank and individual with lower rank is selected. Otherwise, the individual with greater crowding distance is preferred if both the individuals belong to the same rank. In each generation, new individuals are generated through simulated binary crossover and polynomial mutation operations on selected parents. The implementing process for NSGA II is shown as the following pseudo-codes:

Randomly initialize the population $P(0)$ of size $N$; 
Fast non-domination sorting on $P(0)$;

For every generation $t$

Select a parent population $P p(t)$ from $P(t)$ using a binary tournament selection;

Create a child population $P c(t)$ from $P p(t)$ through crossover and mutation operators;

Combine $P(t)$ and $P c(t)$ into an intermediate population $P i(t)$

Fast non-domination sorting on $P i(t)$

Place the best $N$ individuals from $P i(t)$ to $P(t+1)$

End loop

Handling constraints is one of the main issues with genetic algorithms, and many approaches have been proposed, including penalty functions, separation of objectives and constraints, repair algorithms, and hybrid methods (Coello, 2002). For the control optimization problem in this paper, the control parameters are constrained by lower and upper bounds, so NSGA II only needs to handle box constraints, simply by sampling within the intervals.

\section{Control objectives}

One of the main advantages of representing the urban wastewater system as a holistic system lies in the ability to evaluate the performance of the system directly with regard to receiving water quality indicators, rather than by reference to surrogate criteria such as CSO discharge frequency/volume or treatment plant effluent quality (Butler and Schütze, 2005). This is of significant importance because of the fact that no close correlations exist between such surrogate criteria and water quality indicators (Lau et al., 2002). For example, the reduction of overflow volume is not directly linked to an increase of the oxygen concentration in the receiving water (Rauch and Harremoës,1998; Rauch and Harremoës, 1999).

There is a wide range of water quality indicators that can be used to develop control strategies, depending on the real situation of the river and the aim of control. Based on Schütze (1998), the possible indicators include:

- $\quad$ Minimum DO concentration in the river (DO-M); 
- Duration of DO concentration in the river being lower than a predefined critical threshold $(\%$ of the total simulation period) (DO-DU);

- Maximum concentration of ammonium in the river (AMM-M);

- Duration of the ammonium concentration being higher than a predefined critical threshold (\% of the total simulation period) (AMM-DU).

In addition to the water quality parameters mentioned above, cost is another criterion which has a significant impact on decision making for a control strategy. Here consumed energy for pumping and aeration is considered as a cost objective. Pumping energy is estimated using

$$
P=\frac{\rho g Q\left(H_{1}+H_{2}\right)}{\eta}
$$

in which $P$ is power consumed, $\rho$ is the density of pumped liquid, $Q$ the flow rate, $H_{1}$ the geodetic lifting height, $\mathrm{H}_{2}$ the height of frictional losses, and $\eta$ the efficiency of the pump.

Hence in terms of the ultimate aim of optimal control, that is, to maximize the performance of the urban wastewater system, the objective of any optimization algorithm would be to optimize all or a combination of these indicators:

- Maximize DO-M;

- Minimize DO-DU;

- Minimize AMM-M;

- Minimize AMM-DU;

- Minimize $P$;

- Maximize the combination of DO-M and DO-DU (denoted as DO-COM) where

$$
f_{D O-C O M}=\left\{\begin{array}{c}
f_{D O-M}, \quad \text { if } f_{D O-M} \geq D O_{t} \\
D O_{t}\left(1-f_{D O-D U} / 100\right), \quad \text { otherwise }
\end{array}\right.
$$

where $f_{D O-C O M}$ is the value of the combined objective, $f_{D O-M}$ the value of the DO-M objective, $f_{D O-D U}$ the value of the DO-DU objective, and $\mathrm{DO}_{t}$ a predefined threshold of critical DO concentration.

- Minimize the combination of AMM-M and AMM-DU (denoted as AMM-COM) where 


$$
f_{A M M-C O M}=\left\{\begin{array}{c}
f_{A M M-M}, \quad \text { if } f_{A M M-M} \leq A M M_{t} \\
A M M_{t}\left(1+f_{A M M-D U} / 100\right), \quad \text { otherwise }
\end{array}\right.
$$

where $f_{A M M-C O M}$ is the value of AMM-COM, $f_{A M M-M}$ the value of the AMM-M objective,

$f_{A M M-D U}$ the value of the AMM-DU objective, and $\mathrm{AMM}_{t}$ a predefined threshold of ammonium concentration.

According to the definition of DO-COM, in the situation where minimum DO concentration is above the given critical threshold, the objective is reduced to DO-M, while the DO concentration drops below the threshold, the objective measures the duration of breaching the threshold because it becomes more concerned in terms of protection of aquatic life. This formalisation was first used for strategy ranking (Reda, 1996), but Schutze (1998) used this objective in single objective real time control in an effort to include information about minimum concentrations or durations of critical concentrations in one single variable. A $4 \mathrm{mg} / \mathrm{l}$ threshold is chosen for both the $\mathrm{DO}$ and ammonium concentrations in the case study according to the intermittent standards for protection of aquatic life given in the Urban Pollution Management Manual (FWR, 1994).

\section{Case study}

The integrated case study used was originally defined by Schütze (1998) and has been studied using SYNOPSIS for various purposes, including real time control optimization (Schütze et al. 2002b; Butler and Schütze, 2005), control potential analysis (Zacharof et al., 2004) and control objective analysis (Lau et al., 2002). It consists of a sewer system and treatment plant based on real systems to some extent and a hypothetical river system. The catchment is shown in Figure 1.

The sewer system originated from an example (ATV, 1992) and was adapted to match the capacity of the simulated treatment plant. It has seven sub-catchments with a total area of 725.8 ha, and four on-line pass through storage tanks at the downstream end of the linked subcatchments. The storage tanks have a total volume of $13,200 \mathrm{~m}^{3}$. A pump is used to control the outflow of each tank, within the range of two to five times average dry weather flow (DWF). 
The wastewater treatment plant is based on the UK Norwich plant and was adapted to treat an average dry weather flow of $27,500 \mathrm{~m}^{3} / \mathrm{d}$. An additional off line pass through storm tank with a volume of $6750 \mathrm{~m}^{3}$ is located at the inlet to the treatment plant. Filling starts as soon as the maximum inflow rate to the primary clarifier is reached, and emptying is triggered when the inflow drops below the threshold value. The threshold value and emptying rate can be defined by the control strategy.

The river system of concern is $45 \mathrm{~km}$ in length and is equally divided into 45 reaches. The initial and boundary conditions are shown in Table 1. Additional boundary conditions are given by the discharges from CSOs at reach 7, storm tank overflow and treatment plant effluent at reach 10.

Variables from the sewer system and treatment plant, shown in Table 2, are chosen for system control. Different combinations of these variables will result in different control strategies and therefore can be optimized to find the optimal solutions. A base case control strategy taken from Schütze (1998) is used for comparison, and its values and the ranges of the considered variables are shown in Table 2.

Simulation results for the base case scenario are shown in Figures 2a-d. A rainfall event of a total depth of $27 \mathrm{~mm}$ from $7-13^{\text {th }}$ February 1977 is selected for simulation, as shown in Figure $2 a$, based on that used by Schütze (1998). This rainfall event can lead to some CSOs and storm tank usage under the base case control scenario. Figure $2 \mathrm{~b}$ shows the influent to the treatment plant, indicating how the flow is limited by a control variable, i.e., maximum inflow rate to treatment plant. Figures $2 \mathrm{c}$ and $2 \mathrm{~d}$ show $\mathrm{DO}$ and ammonium concentrations at various reaches in the receiving river, including reach 11 located about one kilometer downstream of the treatment plant effluent, reach 20 amongst the middle of reaches, and reach 45 at the downstream of the simulated river.

It can be seen from Figures $2 \mathrm{c}$ and $2 \mathrm{~d}$ that for the base case scenario, $\mathrm{DO}$ concentration at the downstream of the river may drop to $3.58 \mathrm{mg} / \mathrm{l}$, which is below a $4 \mathrm{mg} / \mathrm{l}$ threshold.

As mentioned in Section 4, different control strategies can be devised by considering different performance indicators. In order to objectively evaluate the performance of the system optimized for each indicator combination, a preliminary trial run was performed using the Latin Hypercube Sampling technique for all water quality indicators. From that analysis, it was found that correlation 
between these sets of objectives are not linear or well defined: 1) DO-M and AMM-M, denoted as MOP1; 2) DO-COM and AMM-COM, denoted as MOP2; 3) DO-M, AMM-M, and Cost $P$, denoted as MOP3; 4) DO-COM, AMM-COM, and Cost $P$, denoted as MOP4. Hence, we formulated 4 different multiobjective problems with each one focusing on a set of objectives given above. The aims of investigating all these four combinations of performance indicators are twofold:

(i). Since MOP1 and MOP2 basically measure DO and ammonia concentration but defer in terms of objective function formulation, we would like to investigate the impact of choice of performance indicators on the selection of Pareto optimum solutions;

(ii). MOP1 and MOP3 (and similarly for MOP2 and MOP4) defer in terms of number of objectives considered (i.e. two for MOP1 and three for MOP3). Comparing MOP1 and MOP3 allows us to investigate the advantages (or disadvantages) of adding an additional objective to the problem.

We then applied NSGA II to solve the problems (MOP1 - 4), and the variables were constrained by the intervals in Table 2. So in the case study, NSGA II dealt with box constraints instead of inequality or equality constraints defined in Equations (3) and (4). The following parameter values are used in the optimization process: the generation number is 100 , the population size 100 , the crossover probability 0.9 , and mutation probability 0.1 . The results are shown in the next section.

\section{Results and discussion}

For comparison with MOP1 and MOP3, the DO and ammonium objective values in the optimization results of MOP2 and MOP4 are transformed into DO-M and AMM-M objective values.

\subsection{MOP1 and MOP2}

Figure 3 shows the Pareto optimal solutions from MOP1 and MOP2. There exists a clear tradeoff between the two objectives considered in both cases. Take MOP1 as an example. The solution at the left end of the trade off curve represents a control strategy which can achieve the objective values of $(4.56 \mathrm{mg} / \mathrm{l}, 4.87 \mathrm{mg} / \mathrm{l})$, i.e., the highest (minimum) DO concentration and the lowest (maximum) ammonium concentration in the river. As the ammonium objective improves through all the intermediate solutions down to the solution at the other end with an ammonium 
concentration of $2.92 \mathrm{mg} / \mathrm{l}$, the DO concentration deteriorates to $3.45 \mathrm{mg} / \mathrm{l}$. In other words, it is impossible for a solution to achieve both 'good' DO and ammonium concentrations at the same time. Understanding such underlying tradeoff could help decision makers understand the implications of a control strategy and further gain experience in system control.

None of the solutions in the Pareto front, shown in Figure 3, is absolutely better than any other in terms of the maximum DO and minimum ammonium concentrations. The choice of one solution over the others as a control strategy in practice is subjective, depending on the decision maker's preference. And multicriteria decision making techniques could be used in this situation to guide the decision maker in making rational, transparent decisions. In practice, feasible solutions in the Pareto front could be reduced to a greater extent by taking threshold criteria into consideration. For example, the Urban Pollution Management Manual (FWR, 1994) defines DO and ammonium concentration thresholds that should not be breached beyond a specified frequency. Based on these values, we assume that a $4 \mathrm{mg} / \mathrm{l}$ constraint for $\mathrm{DO}$ and ammonia concentrations is selected in the decision-making process. In Figure 3, the solutions constrained by the $4 \mathrm{mg} / \mathrm{l}$ thresholds should be preferable by decision makers, while solutions in both the tails of the Pareto fronts might be discarded at an early stage of decision making as these solutions breach the thresholds. Once insight into the multi-objective problem has been gained on the whole range of solutions, this kind of prior information could be used to make the optimisation more efficient, i.e., to focus only on parts of the Pareto front.

The performance of the base case control strategy, in terms of DO-M and AMM-M, is also shown in Figure 3. It can be seen that the base case strategy is dominated by many solutions from MOP1, so is outranked by the Pareto front. The same conclusion can be reached when comparing the base case with the Pareto front from MOP2. Therefore system performance, through multiple objective optimization, can be improved in terms of both DO and ammonium concentrations.

Comparing MOP1 and MOP2, there exists a slight gap between the two Pareto fronts, which is probably due to the random characteristics of genetic algorithms and a limited number of generations used in the optimization process. According to Equations (6) and (7), MOP1 and MOP2 have the same objective functions when DO-M is above the $4 \mathrm{mg} / \mathrm{l}$ threshold and AMM-M is 
below. Thus, in this part of the objective function space the two Pareto fronts should be identical in theory. In the other parts of the objective function space, MOP2 actually uses DO-DU and AMMDU as objectives while MOP1 uses DO-M and AMM-M. The different formulations of objectives perhaps is reflected by the slightly wider gap between the other parts of the Pareto fronts (except the left tail part), compared with the part constrained by the thresholds. However, the slight difference in terms of quality of solutions implies in this case that the detailed formulation of the objectives may not be crucial, and this is further verified by comparing MOP3 and MOP4 in Figures 5a-c. This provides quality assurance to the modeler when using multiple-objective optimization method for optimal control.

In order to investigate the behavior of different control strategies in more detail, three control strategies (A, B and C) are selected from Figure 3 for further analysis. Strategies $A$ and $C$ are the two endpoints of the Pareto curve from MOP1 and $B$ is chosen to dominate the base case strategy completely. Figure 4a shows the DO concentration at reach 45 (a critical reach in terms of DO) for each control strategy. And Figure $4 b$ shows the ammonium concentration at reach 11 (a critical reach in terms of ammonium). These tend to confirm the general conclusions drawn from Figure 3.

\subsection{MOP3 and MOP4}

The Pareto optimal solutions from MOP3 and MOP4, shown in Figures 5a-c, are presented in a pair-wise comparison of the three objectives considered. Figure 5a shows a tradeoff between most of the solutions in the left part of the curve, but the solutions in the right part are dominated by others in terms of DO and ammonium objectives. These solutions are included into the Pareto optimal set because they can achieve much better in the objective of energy cost, as seen in Figure 5b. These solutions will be of interest to decision makers who prefer low energy solutions.

Figure $5 \mathrm{~b}$ shows a clear tradeoff between DO and energy objectives. A tradeoff between ammonium and energy objectives can also be observed in the lower part of the curve in Figure $5 \mathrm{c}$, similarly to Figure $5 \mathrm{a}$, the upper consists of dominated solutions in terms of the ammonium and energy objectives, but with a high DO concentration. This leads to a more complex decision making situation because of the relationships between the three objectives. 
Applying the same threshold criteria to Figure 5a, there remain only several preferable solutions in the Pareto front. Compared with MOP1 and MOP2, the preferable solutions are further reduced when one more objective is included in the optimization. Considering threshold criteria, it can be shown in Figure 5a that several such solutions with low energy consumption exist (around $\left.2.35 \times 10^{5} \mathrm{KWH}\right)$. These solutions are much better than the base case scenario with energy consumption of $2.56 \times 10^{5} \mathrm{KWH}$, and better than those Pareto solutions of MOP1 or MOP2 satisfying the same constraint (around $2.38 \times 10^{5} \mathrm{KWH}$ ), shown in Figure 3. This further reinforces the benefit of system control using multiple objective optimization methods.

Though effort was made to reduce the complexity of the integrated model, it still has a high computational demand, which makes it only feasible for offline development of control strategies, given the number of runs required by genetic algorithms. It should be stressed that offline real time control still makes use of the information about current states of the integrated system in the actual control process. Surrogate models such as neural networks have been used to simulate each component of the integrated system, for example, sewer systems (Darsono and Labadie, 2007), wastewater treatment plant (Ráduly et al., 2007) or the integrated system itself, and this provides an effective way to improve computational efficiency for real time control optimization, particularly when these surrogate models are incorporated into genetic algorithms in the optimization process.

\section{Conclusions}

The potential benefits of multiple objective control in integrated urban wastewater system models are demonstrated by a case study. Two water quality indicators in the receiving river and a cost objective are considered in the optimization process. Utilizing the water quality states in the receiving river as control objectives, which is only possible through integrated modelling of the urban wastewater system, represents an advantage in directly measuring the system performance by its final step. Tradeoffs between objectives can be observed in the set of Pareto optimal solutions. This provides decision makers a detailed understanding of the tradeoff relationships when balancing the control objectives to meet various needs in practice. 
A significant improvement in each of the objectives can be observed in comparison with a conventional base case scenario. This further reinforces the benefits to consider multiple objectives in the control process.

The simulation results also show that the multiobjective genetic algorithm employed, NSGA II, is a powerful and effective method for optimal control of the integrated urban wastewater system. For all the defined multiobjective problems with two and three objectives, this method was able to successfully and efficiently derive the Pareto optimal solutions.

\section{Acknowledgements}

This research was supported by the Urban Water Group platform grant at Imperial College London, funded by EPSRC. We would like to thank the reviewer for his useful comments.

\section{References}

ATV-Abwassertechnische Vereinigung e.V., 1992. Richtlinien für die Bemessung und Gestaltung von Regenentlastungsanlagen in Mischwasserkanälen. ATV-Arbeitsblatt A128, Gesellschaft zur Förderung der Abwassertechnik, St. Augustin.

Boomgaard, M.E., Langeveld, J.G., Clemens, F. H. L. R., 2001. Wastewater System Optimization Using Genetic Algorithms. World Water Congress 2001, 111, 498.

Butler, D., Schütze, M., 2005. Integrating simulation models with a view to optimal control of urban wastewater systems. Environmental Modelling \& Software 20, 415-426.

CEC-Council of the European Communities, 2000. Directive 2000/60/EC of the European parliament and of the council of 23 October 2000 establishing a framework for community action in the field of water policy. European Union, Luxembourg.

Coello Coello, C. A., 2002, Theoretical and numerical constraint-handling techniques used with evolutionary algorithms: a survey of the state of the art. Computer Methods in Applied Mechanics and Engineering 8 (2), 1245-1287. 
Coello Coello, C. A., Van Veldhuizen, D. A., Lamont, G. B., 2002. Evolutionary Algorithms for Solving Multi-Objective Problems. Kluwer Academic Publishers.

Copp, J.B. (ed.), 2002. The COST simulation benchmark- description and simulator manual. ISBN 92-894-1658-0, office for official publications of the European communities, Luxumbourg.

Darsono, S., Labadie, J. W, 2007. Neural-optimal control algorithm for real-time regulation of inline storage in combined sewer systems. Environmental modelling and software, 22 (9), 1349 1361.

Deb, K., Pratap, A, Agarwal, S., Meyarivan, T. 2002. A fast and elitist multi-objective genetic algorithm: NSGA-II. IEEE Transaction on Evolutionary Computation 6 (2) 181-197.

Deb, K., 2001. Multi-objective optimization using evolutionary algorithms. New York, NY: John Wiley and Sons Ltd.

FWR, 1994. Urban pollution management manual. Foundation for Water Research, Marlow.

Henze, M. Grady, C.P.L., Gujer, W. Marais, G.v.R., Matsuo, T., 1986. Activated sludge model No.1. LAWQ Scientific and Technical report 1, IAWQ, London, UK.

Huber, W.C., Dickinson, R.E., 1988. Storm Water Management Model. User's Manual Version IV, US Environmental Protection Agency.

IFAK, 2005. SIMBA 5.0: simulation of wastewater systems, user's guide. Institut für Automation und Kommunikation e.V. Magdeburg, Germany.

ITWH, (1995). Mikrocomputer in der Stadtentwässerung-Mischwasserentlastungen, Teil I, KOSIM, Version 4.1, Programmdokumentation, Institut für technisch-wissenschaftliche Hydrologie, Hannover.

Khu, S-T., di Pierro, F, Savic, D., Djordjevic, S., Walters, G. A., 2006. Incorporating spatial and temporal information for urban drainage model calibration: an approach using Preference ordering genetic algorithm. Advances in Water Resources 29(8) 1168-1181.

Lau, J., Butler, D., Schütze, M., 2002. Is combined sewer overflow spill frequency/volume a good indicator of receiving water quality impact. Urban Water 4 (2) 181-189.

Muschalla, D., Schryter, K., Schütze, M., 2006. Multi-objective evolutionary algorithms in the field of urban drainage. Seventh International Conference on Hydroinformatics, Nice. 
Paulsen, O. 1986. Kontinuierliche simulation von abflüssen und schmutzfrachten in der trennentwässerung. Mitteilungen des Institutes für Wasserwirtschaft, Universität Hannover. 62.

Ráduly, B. Gernaey, K.V, Capodaglio, A.G., Mikkelsen, P.S. and Henze, M., 2007. Artificial neural networks for rapid WWTP performance evaluation: Methodology and case study. Environmental Modelling \& Software, 22 ( 8), 1208-1216.

Rauch, W., Aalderink, H., Krebs, P., Schilling, W., Vanrolleghem, P., 1998. Requirements for integrated wastewater models-driven by receiving water objectives. Water Science and Technology 38 (11) 97-104.

Rauch, W., Bertrand-Krajewski, J.-L., Krebs, P., Mark, O., Schilling, W., Schütze, M., Vanrolleghem, P.A., 2002. Mathematical modelling of integrated urban drainage systems. Water Science and Technology 45 (3) 81-94.

Rauch, W., Harremoës, P., 1998. Correlation of combined sewer overflow reduction due to realtime control and resulting effect on the oxygen concentration in the river. Water Science and Technology 37 (12) 69-76.

Rauch, W., Harremoës, P., 1999a. Genetic algorithms in real time control applied to minimize transient pollution from urban wastewater systems. Water Research 33 (5) 1265-1277.

Rauch, W., Harremoës, P., 1999b. On the potential of genetic algorithms in urban drainage modelling. Urban Water 1 79-89.

Reda, A., 1996. Simulation and control of stormwater impacts on river water quality. PhD thesis. Department of Civil Engineering; Imperial College of Science, Technology and Medicine, London.

Savic, D., Khu S,-T., 2005. Applications of evolutionary computing in hydrological sciences. In Anderson M.G. ed., Encyclopedia of Hydrological sciences. J. wiley publisher.

Schütze, M., 1998. Integrated Simulation and Optimum Control of the Urban Wastewater System. PhD thesis. Department of Civil Engineering; Imperial College of Science, Technology and Medicine, University of London, London.

Schütze, M., Butler, D., Beck, M.B, 2002a. Modelling simulation and control of urban wastewater systems, Springer. 
Schütze, M., To, T.B., Jumar, U., Butler, D., 2002b. Multi-objective control of urban wastewater systems, International Federation of Automatic control 15th IFAC World Congress, Barcelona, Spain, 2002.

Schütze, M., Campisano, A., Colas, H., Schilling, W., Vanrolleghem, P.A., 2004. Real time control of urban wastewater systems—where do we stand today? Journal of Hydrology 299 (3-4) 335348.

Takács, I., Patry, G.G., Nolasco, D., 1991. A dynamic model of the clarification thickening process. Water Research 25 (10) 1263-1271.

Vanrolleghem, P.A., Benedetti, L., Meirlaen, J., 2005. Modelling and real-time control of the integrated urban wastewater system. Environmental Modelling \& Software 20 (4) 427-442.

Zacharof, A.I., Butler, D., Schütze, M., Beck, M.B., 2004. Screening for real-time control potential of urban wastewater systems. Journal of Hydrology 299 349-362. 


\section{Figures}

Fig. 1. Schematic representation of the integrated urban wastewater system

Fig. 2a. Rainfall series for simulation

Fig. 2b. Influent to treatment plant

Fig. 2c. DO concentration at various reaches

Fig. 2d. Ammonium concentration at various reaches

Fig. 3. Pareto optimal solutions from MOP1 and MOP2.

Fig. 4a. DO concentration at reach 45 for strategies A, B, C in Fig. 3.

Fig. 4b. Ammonium concentration at reach 11 for strategies A, B, C in Fig. 3.

Fig. 5a-c. Two dimensional plots for Pareto optimal solutions from MOP3 and MOP4.

\section{Tables}

Table 1 Initial and upstream boundary conditions for the river

Table 2 Base case values and ranges for control variables 


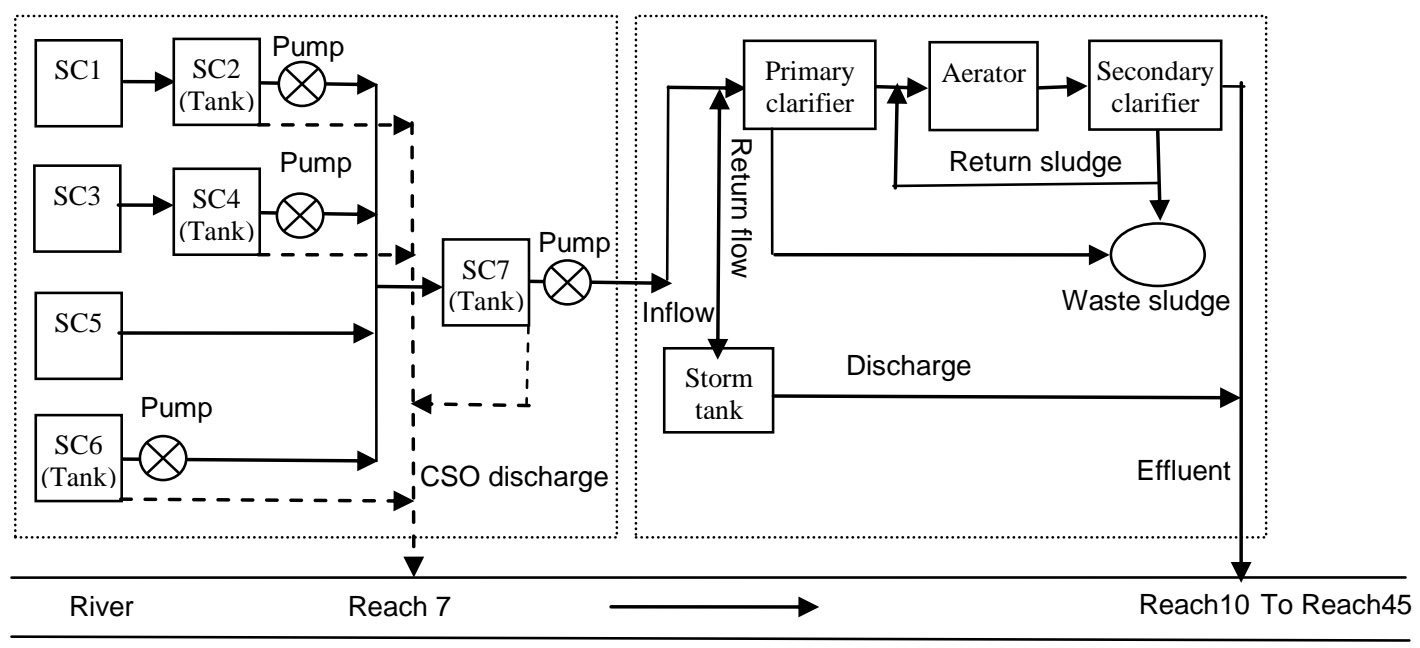

Fig. 1. Schematic representation of the integrated urban wastewater system. SCx represents the xth sub-catchment, and the dash lines show CSO discharge from the storage tanks, located at the downstream of SC2, 4, 6 and 7. 


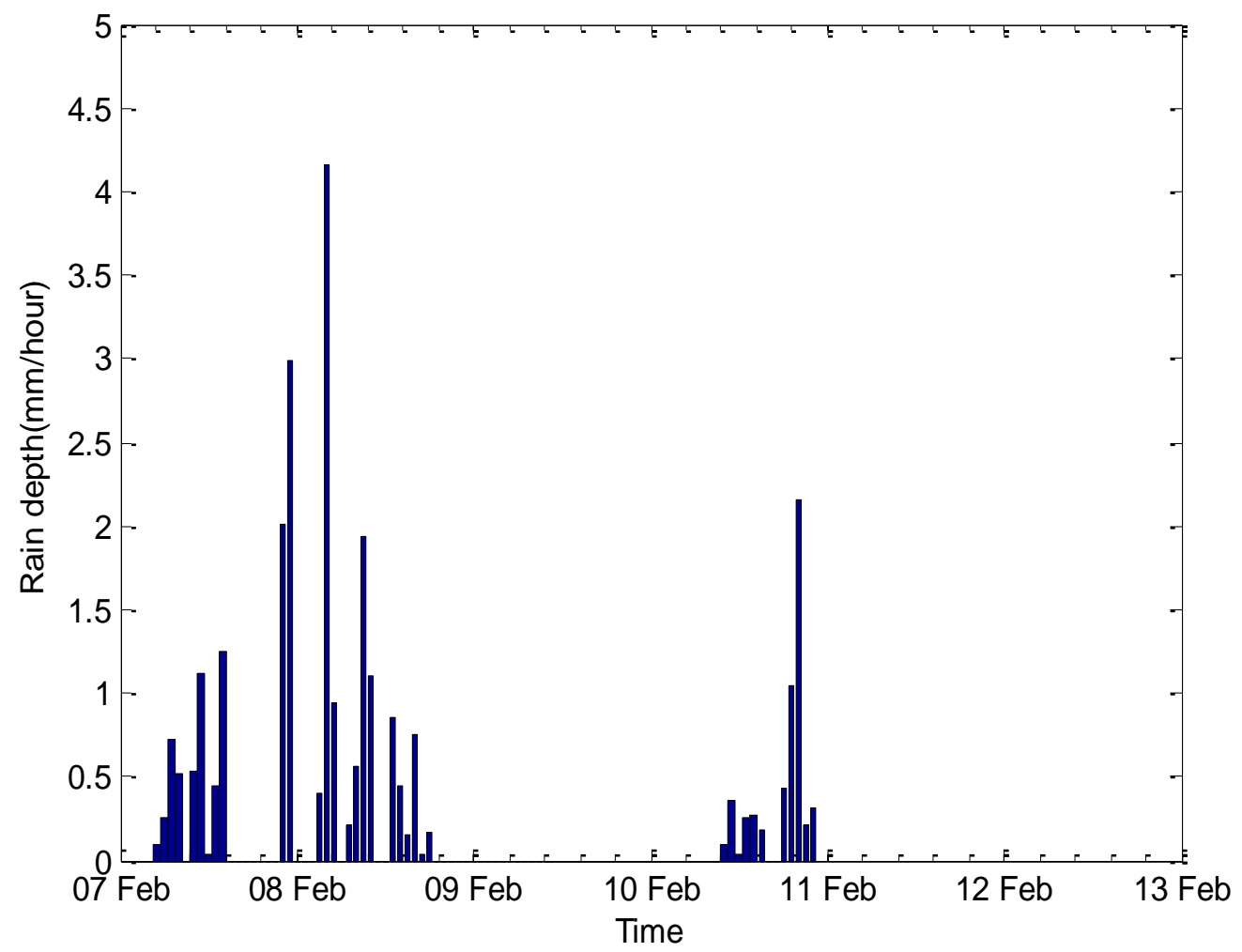

Fig. 2a. Rainfall series for simulation 


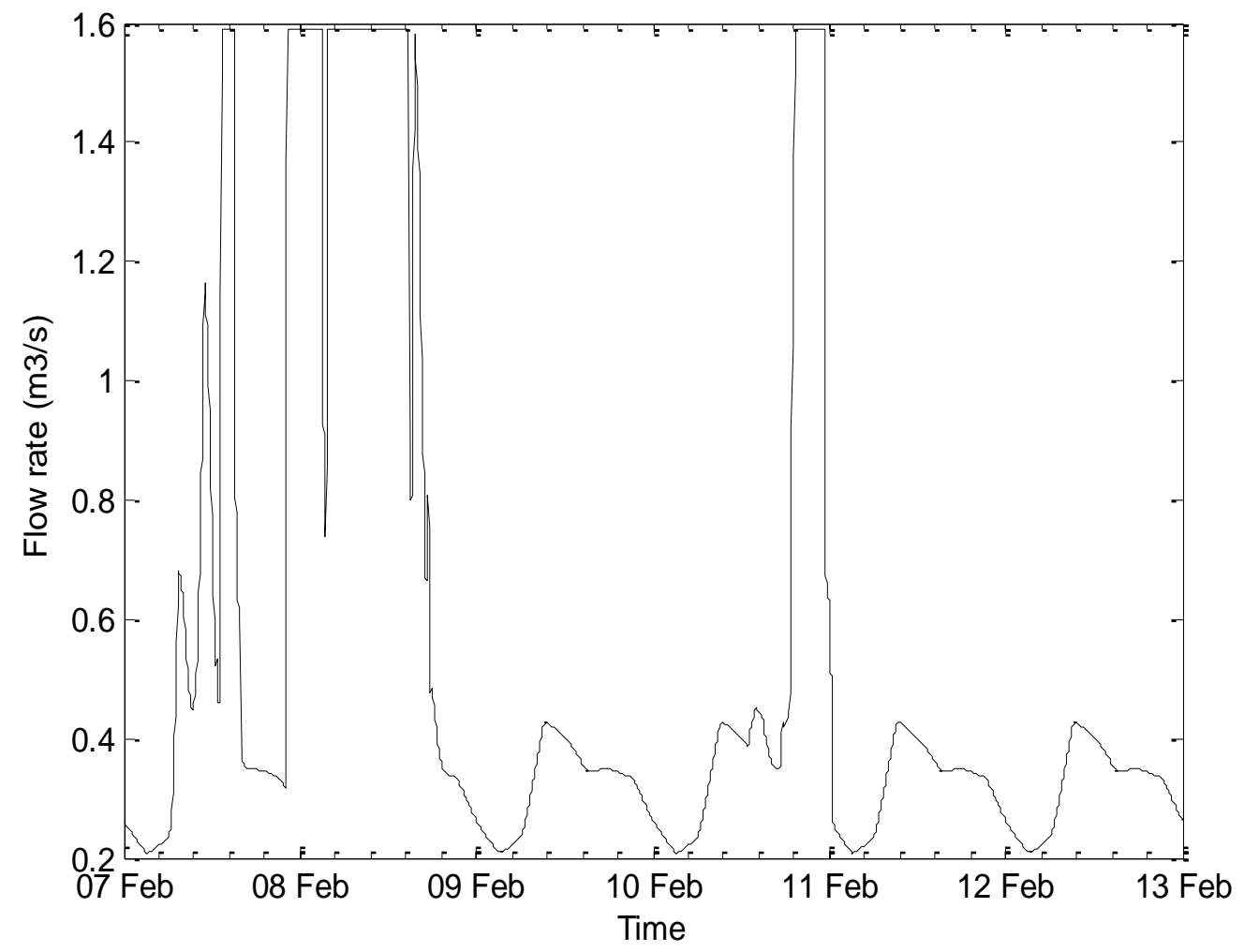

Fig. 2b. Influent to treatment plant 


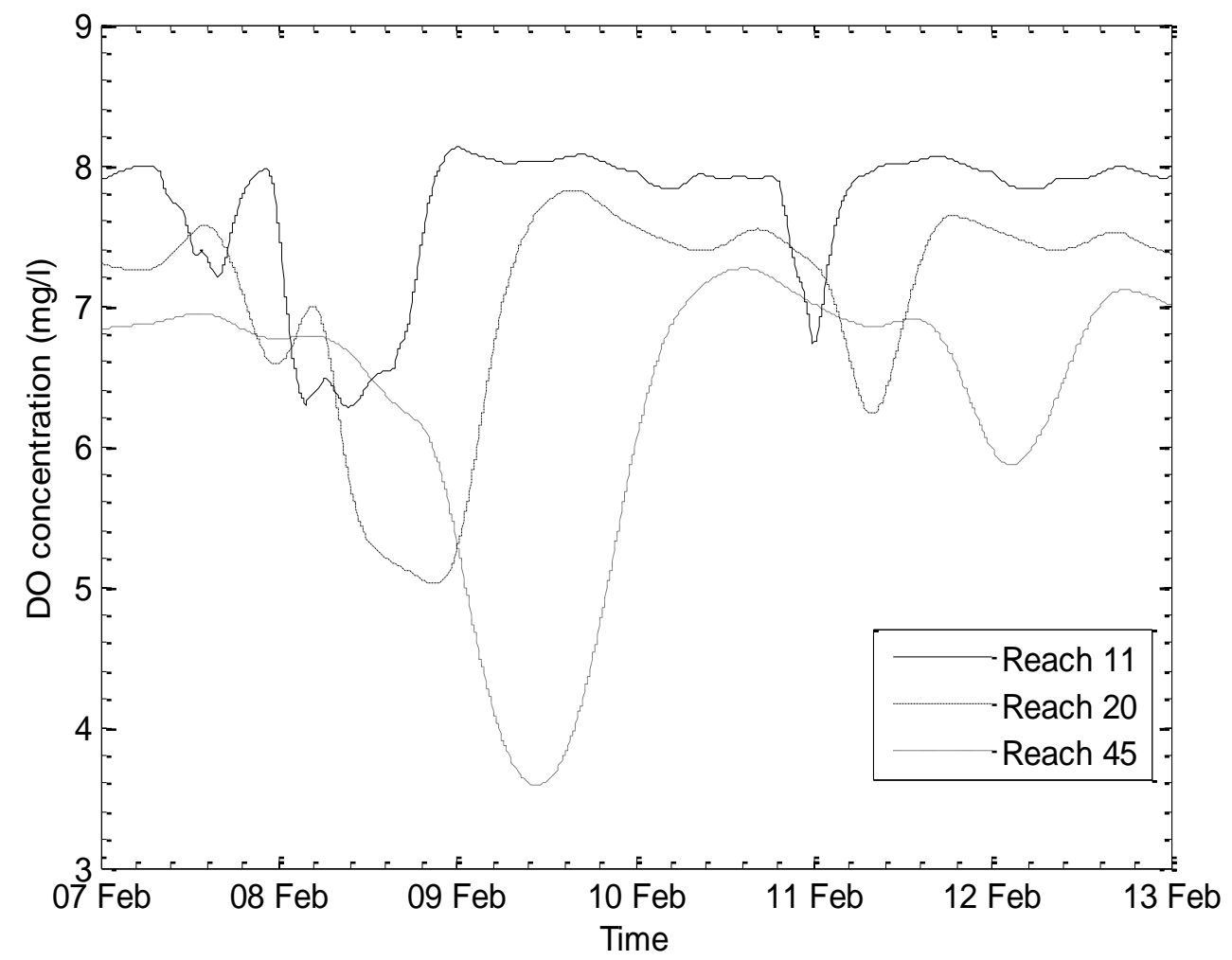

Fig. 2c. DO concentration at various reaches 


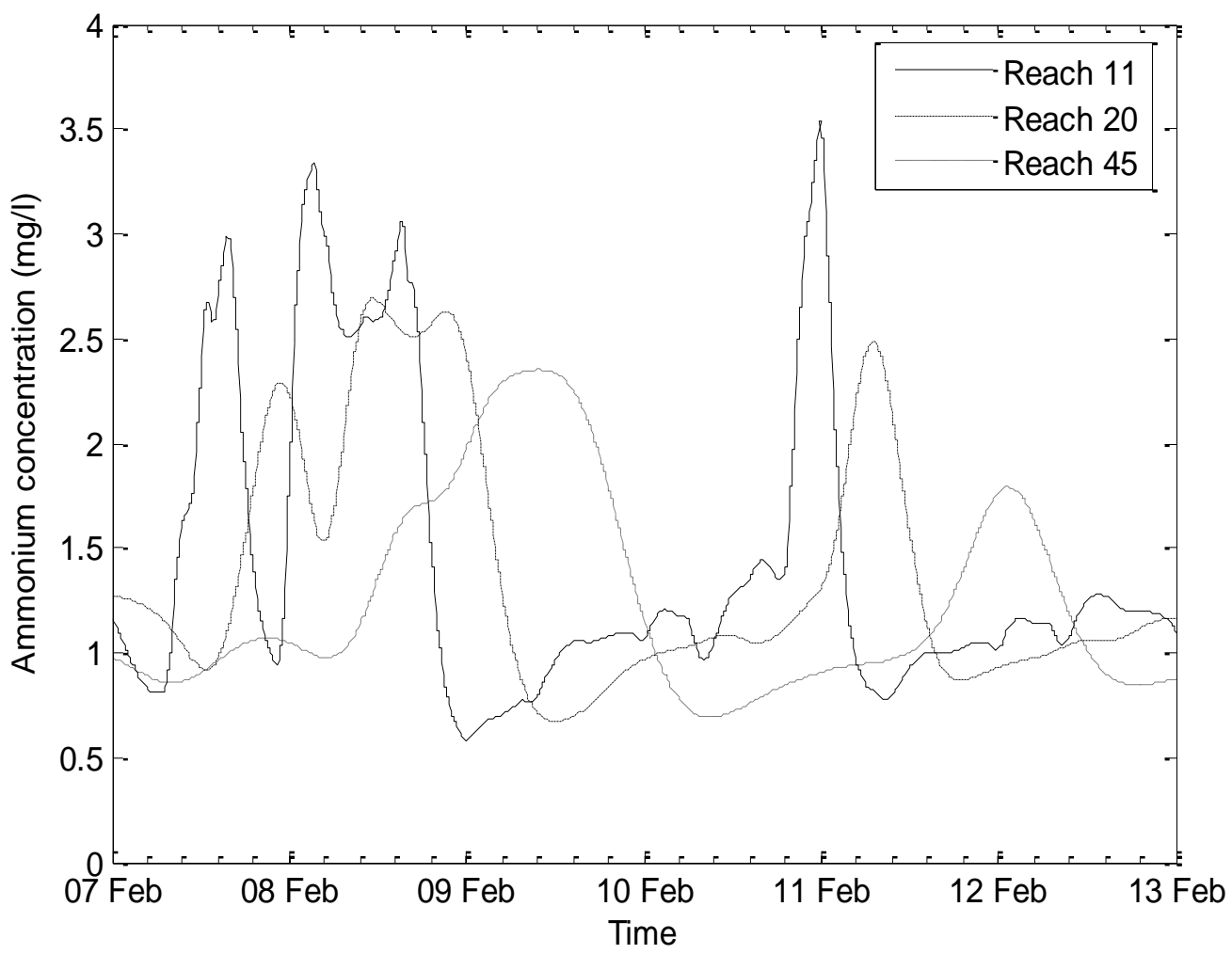

Fig. 2d. Ammonium concentration at various reaches 


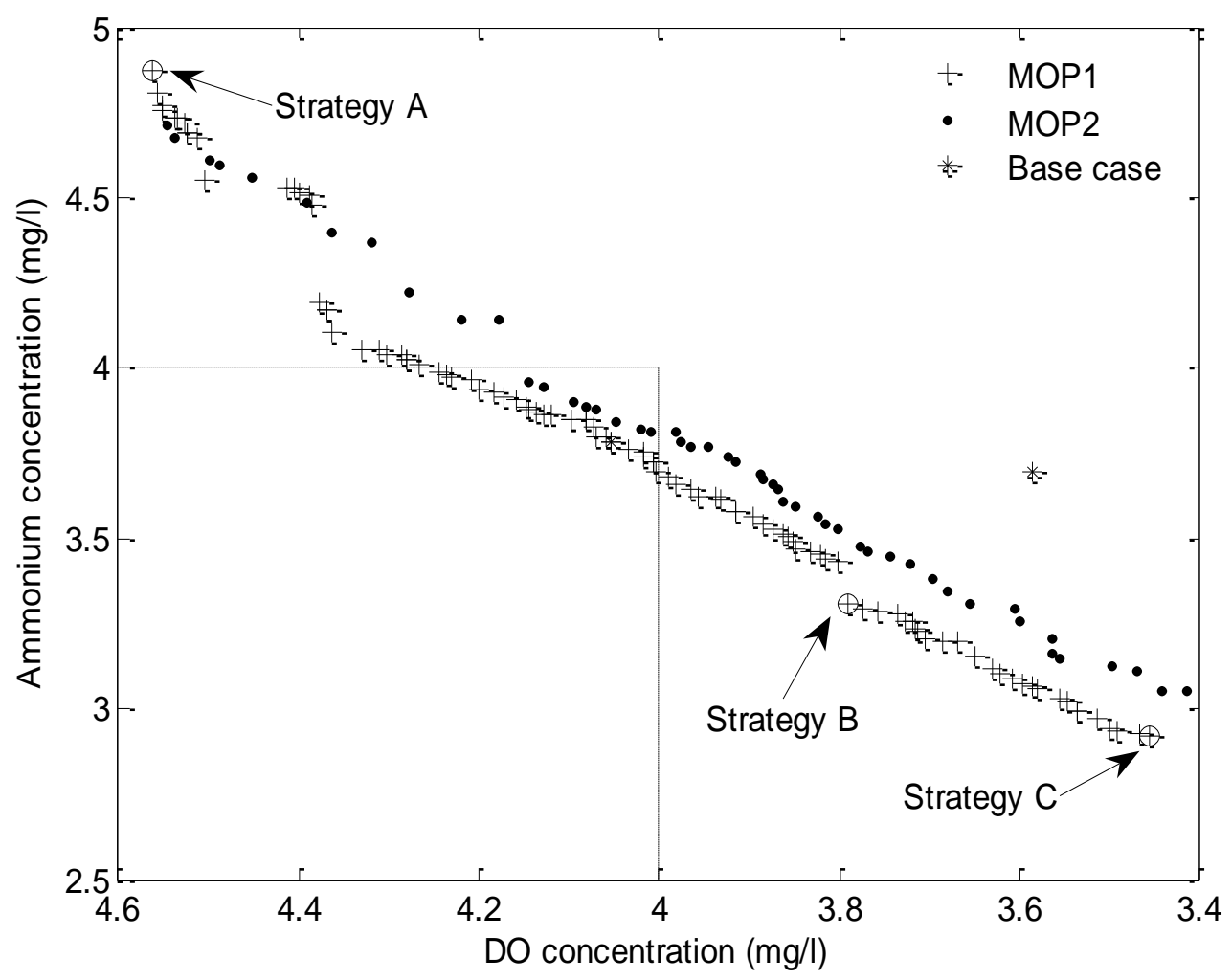

Fig. 3. Pareto optimal solutions from MOP1 and 2. 


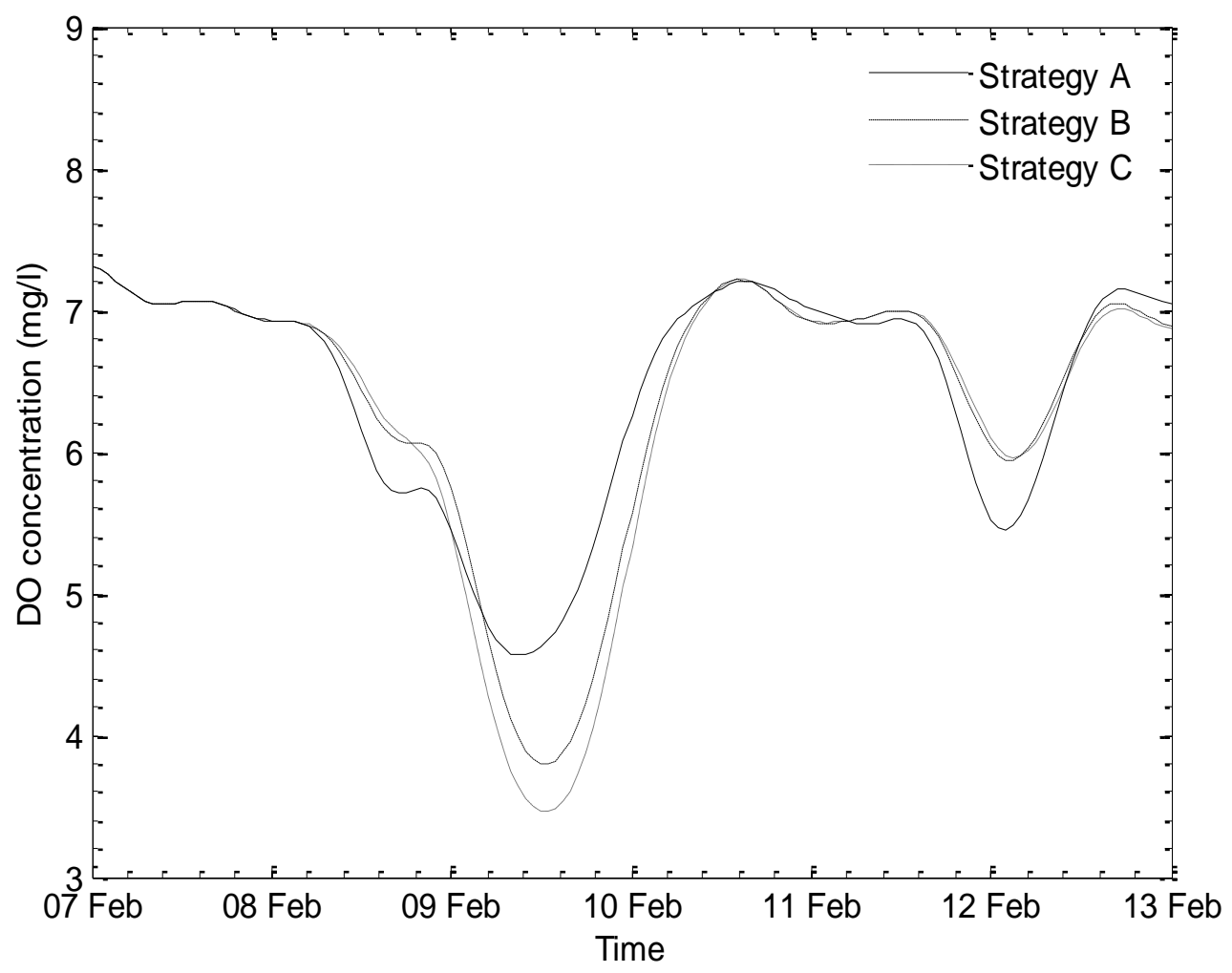

Fig. 4a. DO concentration at reach 45 for strategies A, B, C in Fig. 3. 


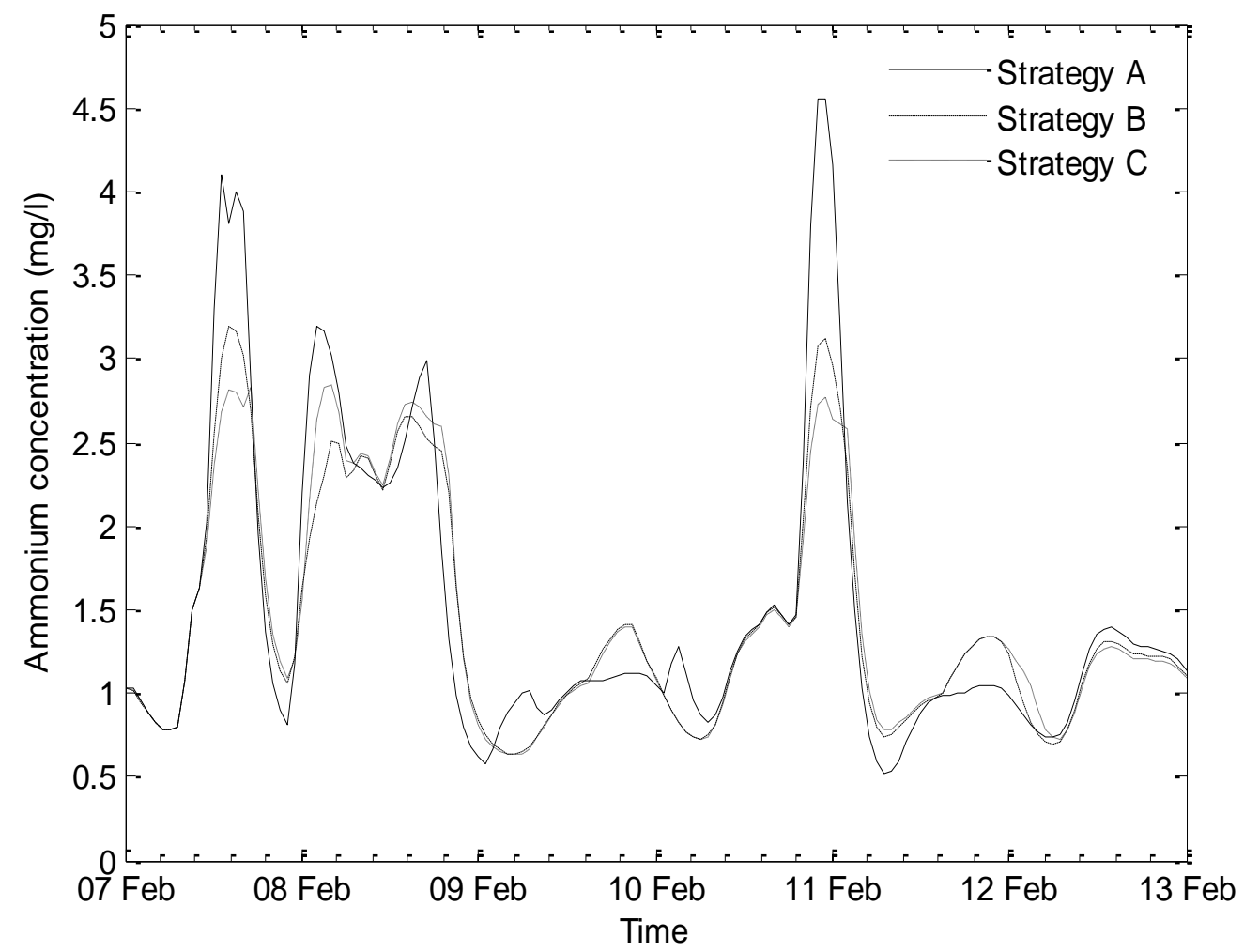

Fig. 4b. Ammonium concentration at reach 11 for strategies A, B, C in Fig. 3. 


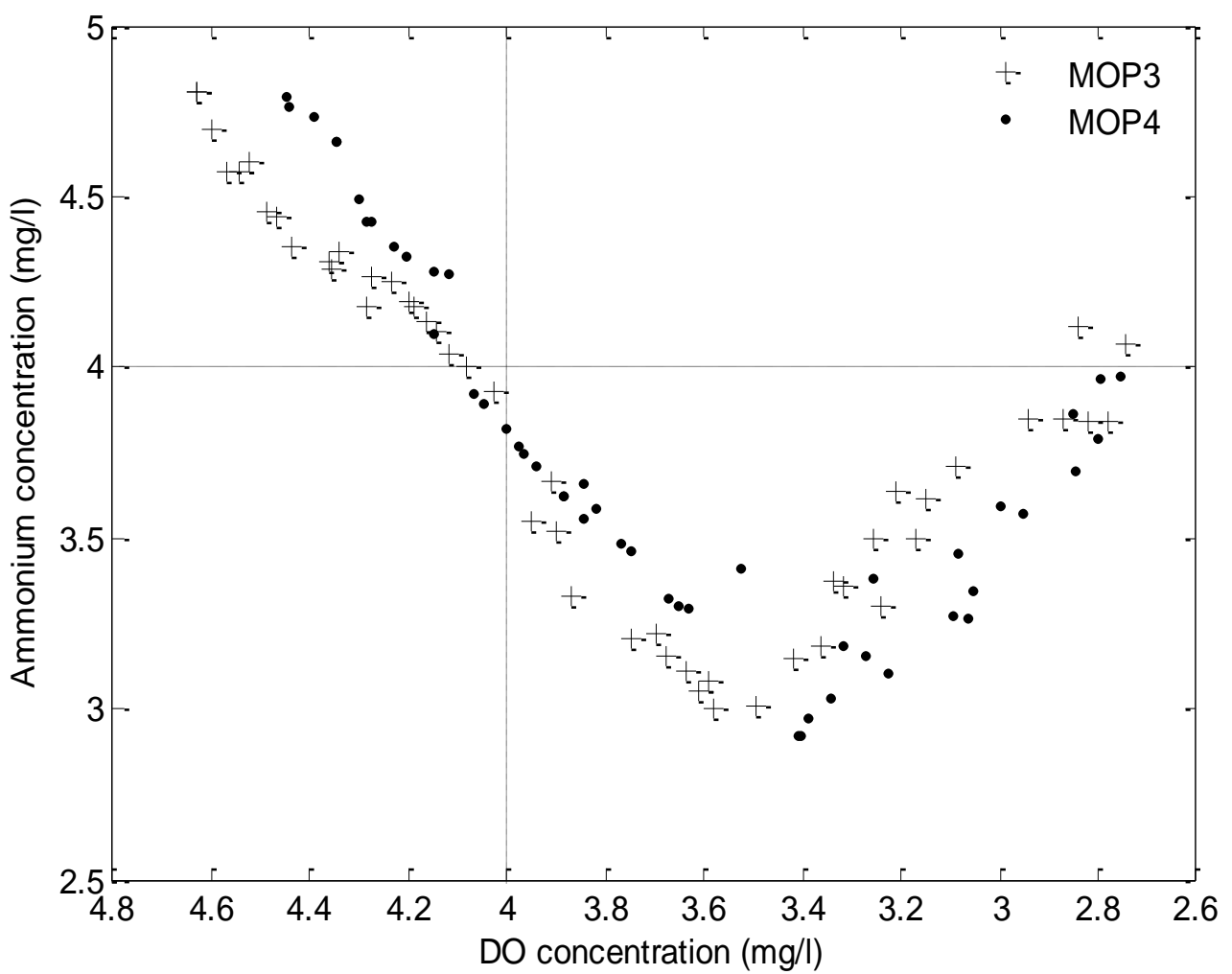

Fig. 5a. DO and ammonium objectives 


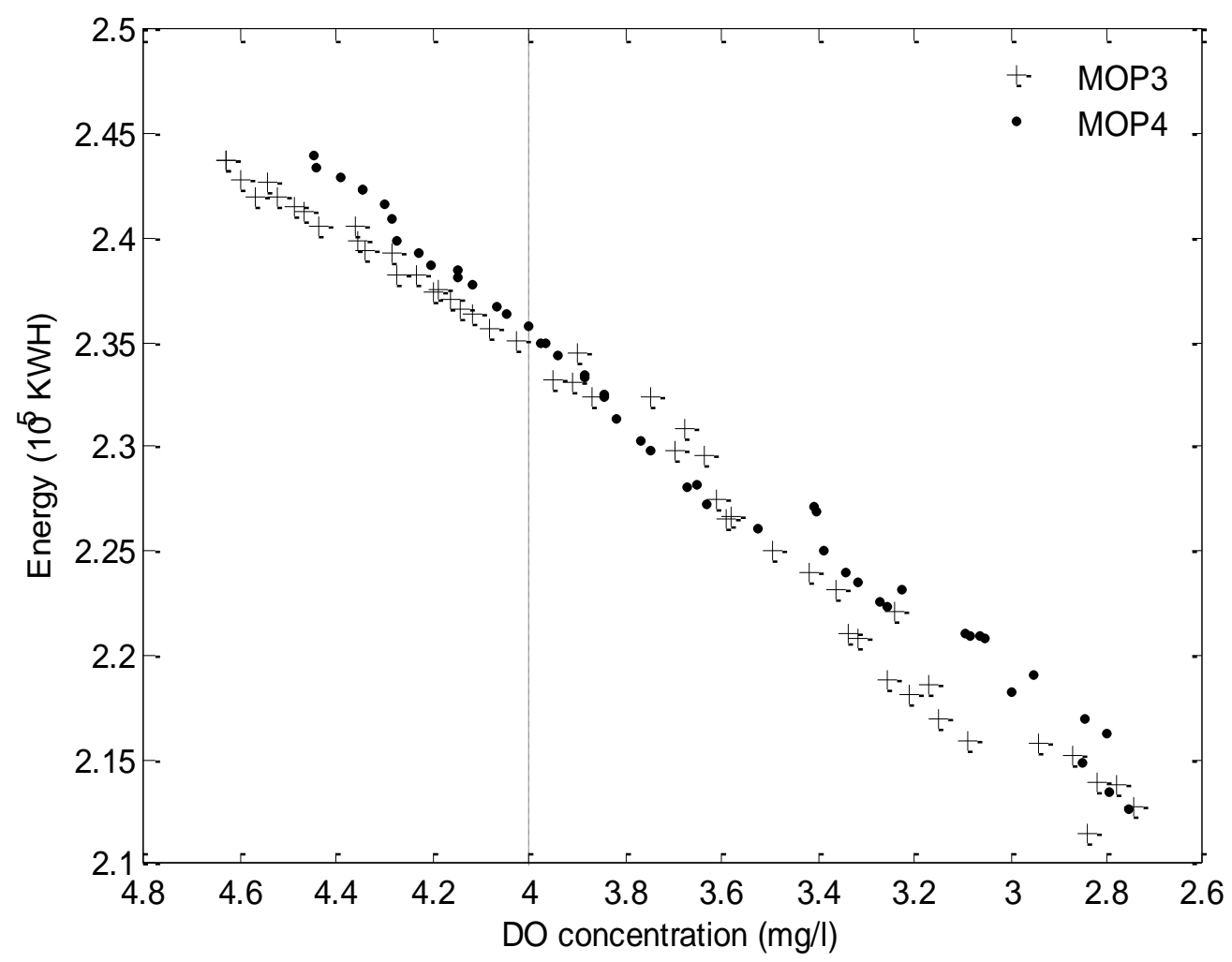

Fig. 5b. DO and energy objectives 


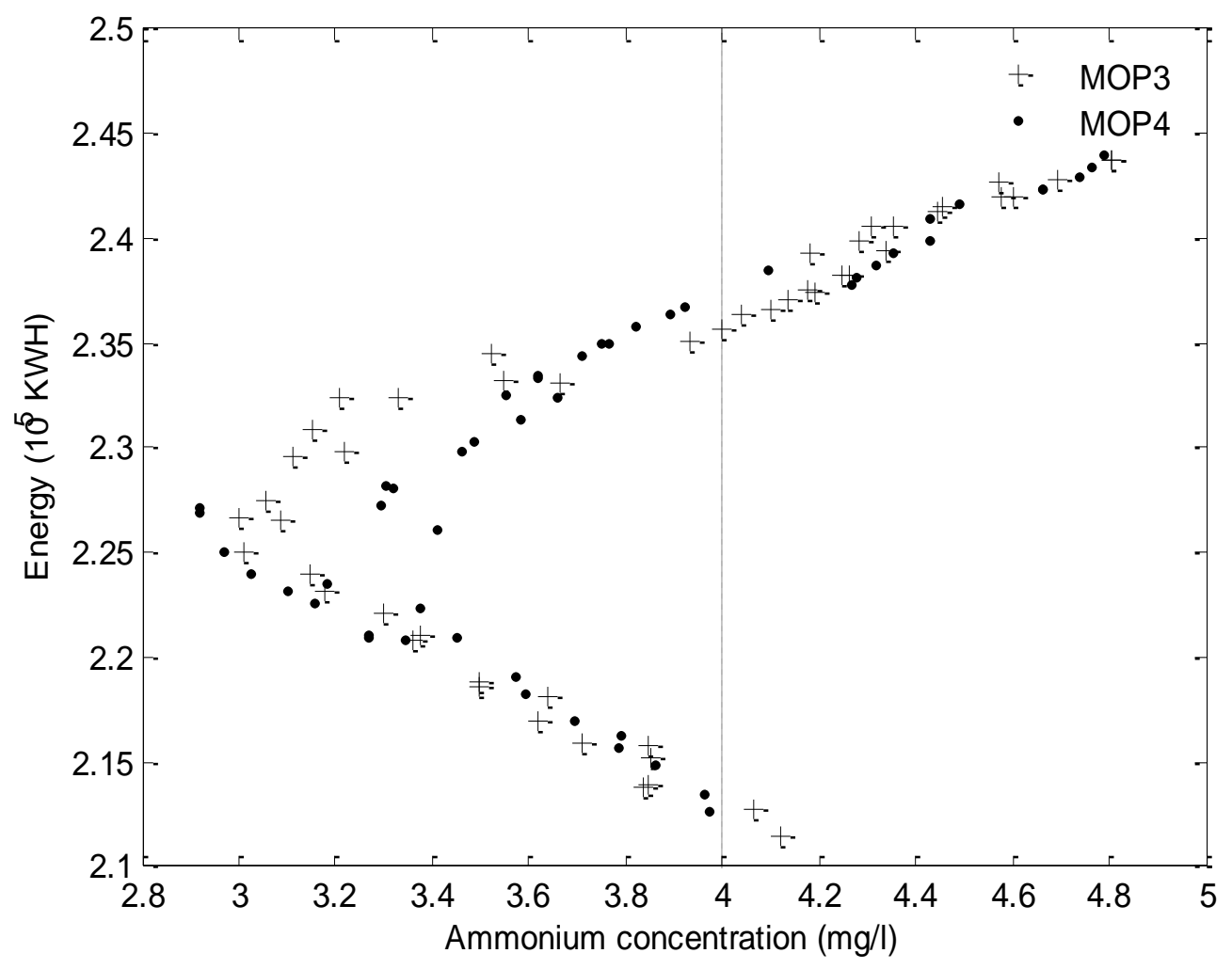

Fig. 5c. Ammonium and energy objectives 
Table 1 Initial and upstream boundary conditions for the river (Schütze, 1998)

\begin{tabular}{ll}
\hline \multicolumn{1}{c}{ Variable } & Initial values \\
\hline Flow & $1.5 \mathrm{~m}^{3} / \mathrm{s}$ \\
$\mathrm{NH}_{3}$ & $0.09 \mathrm{mg} / \mathrm{l}$ \\
BODr & 0 \\
BODs & $1.80 \mathrm{mg} / \mathrm{l}$ \\
DO & $9.00 \mathrm{mg} / \mathrm{l}$ \\
\hline
\end{tabular}


Table 2 Base case values and ranges for control variables (Schütze, 1998)

\begin{tabular}{lll}
\hline \multicolumn{1}{l}{ Description } & Base case values & Variable ranges \\
\hline $\begin{array}{l}\text { The maximum outflow rate of the storage tank } \\
\text { linked to sub-catchment } 7 \text { (DWF) }\end{array}$ & {$[3,8]$} \\
Maximum inflow rate to treatment plant (DWF) & 3 & {$[2,5]$} \\
Threshold triggering emptying the storm tank $\left(\mathrm{m}^{3} / \mathrm{s}\right)$ & 0.28 & {$[0.19,0.36]$} \\
Emptying flow rate of storm tank $\left(\mathrm{m}^{3} / \mathrm{s}\right)$ & 0.14 & {$[0.08,0.28]$} \\
Return activated sludge rate $\left(\mathrm{m}^{3} / \mathrm{s}\right)$ & 0.17 & {$[0.08,0.28]$} \\
\hline
\end{tabular}

